\title{
THE EFFECT OF ANTICOAGULANT TYPES ON THE IN VITRO ANALYSIS OF CLOPIDOGREL IN HUMAN PLASMA USING LIQUID CHROMATOGRAPHY TANDEM-MASS SPECTROMETRY
}

\author{
YAHDIANA HARAHAP*, ANISA MAULIDINA, DELLY RAMADON \\ Faculty of Pharmacy, Universitas Indonesia, Depok 16424, Indonesia. Email: yahdiana03@yahoo.com
}

Received: 08 June 2018, Revised and Accepted: 09 August 2018

\section{ABSTRACT}

Objective: The aim of this study was to optimize and validate a plasma clopidogrel analysis method using liquid chromatography tandem-mass spectrometry.

Methods: Plasma samples were analyzed using a BEH C18 column $(1.7 \mu \mathrm{m} ; 100 \mathrm{~mm} \times 2.1 \mathrm{~mm})$, the mobile phase was $0.1 \%$ formic acid in acetonitrile $(30: 70, v / v)$. The flow rate was $0.2 \mathrm{~mL} / \mathrm{min}$, with a column temperature set to $35^{\circ} \mathrm{C}$, an injection volume of $5 \mu \mathrm{L}$, an analysis time of $4 \mathrm{~min}$, and irbesartan as the internal standard. Aliquots were obtained by liquid-liquid extraction using ammonium acetate and diethyl ether. The stability and peak area ratio of the respective plasma area responses were evaluated using ANOVA.

Results: No significant differences ( $>>0.05)$ were observed between anticoagulants regarding analyte stability. However, the peak area ratio showed significant differences $(\mathrm{p}<0.05)$ between the anticoagulants. The accuracy and precision of the analysis with citrate, heparin, and ethylenediaminetetraacetic acid (EDTA) plasma met the quality requirements, and a linear calibration curve was created with concentrations ranging from 0.02 to $5.0 \mathrm{ng} / \mathrm{mL}$.

Conclusion: The results showed that improved analysis of clopidogrel was achieved using citrate or heparin plasma compared with EDTA plasma.

Keywords: Citrate, Clopidogrel, Ethylenediaminetetraacetic acid, Heparin, Liquid chromatography tandem-mass spectrometry.

(C) 2018 The Authors. Published by Innovare Academic Sciences Pvt Ltd. This is an open access article under the CC BY license (http://creativecommons. org/licenses/by/4. 0/) DOI: http://dx.doi.org/10.22159/ijap.2018.v10s1.87

\section{INTRODUCTION}

Clopidogrel is one of the slowest-onset action prodrugs (Brunton, Lazo, and Parker, 2006) and is the drug of choice for antiplatelet agents [1]. Clopidogrel is also used as secondary prevention of cerebral and cerebrovascular infarction in patients who do not tolerate acetylsalicylic acid or experience new attacks while using acetylsalicylic acid [2]. Clopidogrel is adenosine diphosphate receptor antagonist and irreversibly inhibits platelet function [3].

The maximum concentration of clopidogrel in plasma is reportedly very low, that is, $7921.49 \pm 3921.39 \mathrm{pg} / \mathrm{mL}$ [4]. Thus, developing accurate analytical methods for clopidogrel are important, particularly for the examination and monitoring of clopidogrel levels in plasma, which is the most common biological matrix for the analysis of drugs in the body. Plasma is obtained from blood samples with the use of anticoagulants. Thus, an important factor when obtaining plasma is the type of anticoagulant used and studies often use different anticoagulants, making comparisons between analytical methods difficult [5,6]. The ethylenediaminetetraacetic acid (EDTA), heparin, and citrate are anticoagulants often used in drug [5].

The anticoagulants themselves are generally present in the blood collection tube at a concentration sufficient to inhibit blood clotting, as the use of excess concentrations may lead to undesirable effects during bioanalysis [7]. However, the use of anticoagulants can lead to errors during the analysis of certain drugs [8]. Thus, selecting the appropriate anticoagulant during drug analysis serves to provide many benefits, including minimizing interferences and improving the stability of the drug and/or its metabolites [7]. Indeed, differences between anticoagulants have been known to affect the measurement of small molecules [9], metabolic profiles [6,10], and clinical parameters of the drug being analyzed [11-13]. In addition, some of the ions (e.g., $\mathrm{Na}$ + or $\mathrm{K}+$ ) present in the plasma anticoagulant such as Na-citrate and
K-EDTA may cause ion suppression or ion enhancement of the drug and its metabolites, which may affect analysis [6]. Moreover, for plasma drug analysis, a selective and sensitive bioanalysis method is required because the plasma level of clopidogrel is very low [14]. Ultra-highperformance liquid chromatography tandem-mass spectrometry (UHPLC-MS/MS) with electrospray ionization (ESI) is a specific and sensitive method of analysis that has become the standard for the measurement of drugs, metabolites and endogenous compounds in biological matrices [15].

This study was conducted to evaluate the commonly used anticoagulant types, namely, citrate, heparin, and EDTA, on parameters such as stability, recovery, matrix effect, and area response of the analyte.

\section{MATERIALS AND METHODS}

\section{Materials}

Standard clopidogrel (MSN Laboratories, India) and standard irbesartan (Zhejiang Huahai Pharmaceutical, China) were purchased. Blood was taken directly from healthy subjects, and plasma was obtained using the citrate anticoagulant citrate phosphate dextrose adenine (CPD-A) (Indonesian Red Cross). Reagents were high PLC (HPLC)-grade and included acetonitrile, formic acid, diethyl ether, and ammonium acetate were purchased from Merck.

\section{Tools}

A ultra PLC-MS/MS (UPLC-MS/MS) system (Waters Xevo TQD Triple Quadrupole) consisting of a quaternary solvent manager (Acquity UPLC H-Class), Sample ManagerFTN (Acquity UPLC), a nitrogen generator compressor (PEAK Scientific), a BEH C18 column (Waters Acquity ${ }^{\mathrm{Tm}}$ UPLC $100.0 \mathrm{~mm} \times 2.1 \mathrm{~mm}, 1.7 \mu \mathrm{m}$ ), a triple quadrupole (Xevo TQD) mass analyzer with an ionization source (Zspray ${ }^{\text {TI'}}$ ), data processing software (MassLynx Software), and a computer was used. Other equipment used included vacuum tubes or Vacutainers with K3EDTA anticoagulant 
(Vacuette ${ }^{\circledR}$ ), vacuum tubes with the anticoagulant Li-Heparin (Vacuette ${ }^{\circledR}$ ), blood collection tubes (BD Vacutainer ${ }^{\circledR}$ Beckton, Dickson and Company), analytical scales (Acculab), degasser (Elmasonic S40H), evaporator (Turbovap), centrifuge (Digital Lab Centrifuge), vortex (Maxi Mix II), $\mathrm{pH}$ meter ( $\mathrm{pH}$ Eutech 510 ), refrigerator $\left(4-8^{\circ} \mathrm{C}\right.$ ), freezers $\left(-20^{\circ} \mathrm{C}\right.$ and $\left.-80^{\circ} \mathrm{C}\right)$ (Biomedical Labtech DeepFreeze), Eppendorf tubes (Soccorex), centrifugation tubes, blue tips, yellow tips, and various glassware.

\section{Sample preparation}

The quantitative determination of an analyte and its metabolites in a biological matrix takes place in several stages, including sample storage, sample preparation, separation, identification, and analyte quantification. Sample preparation has three principles: Dissolution of the analyte in a suitable solvent, the removal of any interfering compounds, and the preconcentration of the analyte. Some of the techniques employed include protein precipitation, liquid-liquid extraction, solid phase extraction, and ultrafiltration. In this research, the sample preparation used liquid-liquid extraction using ammonium acetate and diethyl ether.

\section{Chromatography condition}

Chromatographic separation is based on the transfer of a liquid (mobile phase) carrying the analytical mixture through a stationary medium. The interaction difference of the analyte with the surface of the medium results in a difference in retention time between the components of the mixture [16-20]. UPLC differs from other chromatographic methods in the size of the column particles is substantially smaller. With UPLC, the speed of analysis and peak capacity is maximized. In addition, solvents are used less due to the high analysis speeds; thus, UPLC improves efficiency and resolution [21]. Small diameter UPLC diameter particles $(<2.0 \mu \mathrm{m})$ can improve efficacy (Waters, 2016). The instrumentation of a UPLC system is almost the same as LC and generally consists of a solvent reservoir, pump, injector, column, detector, and a data processing system. The bridged ethylene hybrid column (BEH) has the advantage of high retentivity for basic compounds, good peak shape at high $\mathrm{pH}$, universal columns for various compounds, stability at various pHs and can be used for separation at high temperatures $\left(80^{\circ} \mathrm{C}\right)$ [17].

\section{Analysis conditions}

The analytical method used in this study was UPLC. For analysis, was used C18 columns (100 $\mathrm{mm} \times 2.1 \mathrm{~mm}, 1.7 \mu \mathrm{m})$, a $0.1 \%$ formic acid filtration phase in $0.1 \%$ formic acid solution in acetonitrile $(30: 70, v / v)$, as mobile phase isocratic elution at $0.2 \mathrm{~mL} / \mathrm{min}$ flow rate, and a $35^{\circ} \mathrm{C}$ column temperature. MS detection using the ESI positive ionization method and MRM was performed with capillary tubing of $3.5 \mathrm{kV}$, a desecration gas temperature of $400^{\circ} \mathrm{C}$, a dissolved gas flow rate of $800 \mathrm{~L} / \mathrm{h}$. The obtained $\mathrm{m} / \mathrm{z}$ values were the molecular weight of the parent ion and the molecular weight of the productions. The molecular weight value of clopidogrel was $321.8 \mathrm{~g} / \mathrm{mol}$, while that of irbesartan was $428.5 \mathrm{~g} / \mathrm{mol}$, with clopidogrel at $\mathrm{m} / \mathrm{z} 322.086>212.097$ and irbesartan at $\mathrm{m} / \mathrm{z} 429.233>207.131$.

\section{System suitability test}

Clopidogrel and irbesartan standards were carefully weighed and placed into a measuring flask and dissolved with acetonitrile to the measurement limit to obtain a solution of $1 \mathrm{mg} / \mathrm{mL}(1000 \mu \mathrm{g} / \mathrm{mL})$. The two dilutes were diluted again to the concentration of the system suitability test solution and the concentration solution of the calibration curve. A $100 \mathrm{ng} / \mathrm{mL}$ clopidogrel mixture solution was injected (as much as $10.0 \mu \mathrm{L}$ ) into the chromatography system. Injection results were recorded and counted for the return value (\%KV) over five different injections. The calculated parameters were the areas and times of peak analytical and internal retention peaks.

\section{Blood sampling and plasma preparation}

Plasma with the citrate anticoagulant CPD-A was obtained from the Indonesian Red Cross (Palang Merah Indonesia), while blood plasma with the anticoagulants heparin and EDTA was obtained from the separation of blood samples taken from six healthy subjects that gave informed consent. Blood samples were divided into two vacuum tubes, each containing anticoagulants in the form of Li-Heparin and K3EDTA. Blood in each vacuum tube was inverted 10 times to mix the blood with the anticoagulant in the tube. The vacuum tube was centrifuged at $3000 \mathrm{rpm}$ for $10 \mathrm{~min}$ at room temperature. The plasma was then transferred to another clean tube. Plasma was stored in a freezer at $-80^{\circ} \mathrm{C}$ until analysis.

\section{Sample preparation}

A total of $250 \mu \mathrm{L}$ plasma containing clopidogrel of a certain concentration plus $20 \mu \mathrm{L}$ irbesartan $(100 \mathrm{ng} / \mathrm{mL})$ was vortexed for $10 \mathrm{~s}$. Plasma with ammonium acetate $(0.05 \mathrm{M}$; pH 6.8) was vortexed for $10 \mathrm{~s}$. The extraction was performed with the addition of diethyl ether, vortexed, and centrifuged at 13,000 rpm for $10 \mathrm{~min}$. A total of $750 \mu \mathrm{L}$ of the organic phase was separated into clean tubes to evaporate and dry at $60^{\circ} \mathrm{C}$ for $10 \mathrm{~min}$. The obtained residue was reconstituted in $150 \mu \mathrm{L}$ acetonitrile, vortexed for $10 \mathrm{~s}$, sonicated for $10 \mathrm{~s}$, and vortexed again for $10 \mathrm{~s}$. The final solution was transferred to a vial insert and centrifuged at $3000 \mathrm{rpm}$ for $5 \mathrm{~min}$. A total of $5.0 \mu \mathrm{L}$ of the final solution was injected into the chromatographic system.

\section{RESULTS AND DISCUSSION}

The internal standard used irbesartan because it has similar physicochemical properties to clopidogrel, particularly its acidity and solubility; thus, it can be detected and eluted using the same analytical method. The analytical conditions resulted in a good separation between the analyte and the standard with clopidogrel retention of $2.68 \mathrm{~min}$ and an irbesartan retention of $1.16 \mathrm{~min}$. A relatively short run time of 4 min was also considered a good achievement of this method.

Liquid-liquid extraction methods can produce larger analytical areas [12]. In this study, the liquid-liquid extraction method was optimized. Liquid-liquid extraction is best used for analysis using HPLC-MS/MS because it does not damage the column when compared with the protein precipitation method. In addition, the resulting area response is greater due to the drying process, and fewer additional solvents (concentration process) are used. The optimization of the liquid-liquid extraction process conducted in this study was aimed to obtain an efficient and rapid extraction step so as to minimize contamination. However, during liquid-liquid extraction, an emulsion can sometimes form during processing, so the process needs to be repeated. Thus, a full validation was performed on plasma citrate [22].

Comparison of clopidogrel analysis in three types of anticoagulants The different characteristics of each anticoagulant, including their different physicochemistry, plasma $\mathrm{pH}$, and ion type differences, it was necessary to analyze three types of anticoagulants by observing the chromatograms and parameters produced by each plasma, including the recovery value, peak area ratio, the stability of the analytes, and matrix effects.

Comparison of the chromatograms and spectrums for all three plasmas

Observations were made by comparing the plasma chromatograms, which showed no significant differences. Other observations were made by comparing the spectrum of the blanks for each plasma. The matrix effect of the three plasmas showed no significant differences.

\section{Comparison of clopidogrel area responses in all three plasmas}

Based on the data analysis on Peak Area Ratio (PAR), the obtained $\mathrm{p}<0.05$ (Kruskal-Willis) for all concentrations. This showed that there was a significant difference between the PAR values generated from the three types of plasma. Furthermore, further statistical analysis using the Mann-Whitney method for two types of plasma indicated that the ratio of the citrate-heparin plasma at all concentrations has a $p>0.05$, indicating no significant difference. In contrast, plasma citrate-EDTA and heparin-EDTA analysis had a value of $\mathrm{p}<0.05$, meaning that there was a significant difference. 
Table 1: Comparison of clopidogrel analysis in three plasma samples

\begin{tabular}{|c|c|c|c|c|c|c|}
\hline \multirow{2}{*}{\multicolumn{2}{|c|}{ Parameter analysis }} & \multicolumn{3}{|l|}{ Types of plasma } & \multirow[t]{2}{*}{$\mathbf{p}$} & \multirow[t]{2}{*}{ Explanation } \\
\hline & & Citrate & Heparin & EDTA & & \\
\hline \multicolumn{7}{|c|}{ Peak area ratio $( \pm S D)$} \\
\hline 1 & LLOQ & $0.1931 \pm 0.02$ & $0.1833 \pm 0.03$ & $0.1230 \pm 0.01$ & $<0.05$ & Citrate-EDTA; Heparin-EDTA \\
\hline 2 & QCL & $0.2509 \pm 0.02$ & $0.2448 \pm 0.04$ & $0.1635 \pm 0.01$ & $<0.05$ & Citrate-EDTA; Heparin-EDTA \\
\hline 3 & QCM & $2.0008 \pm 0.19$ & $2.1025 \pm 0.35$ & $1.3953 \pm 0.13$ & $<0.05$ & Citrate-EDTA; Heparin-EDTA \\
\hline 4 & $\mathrm{QCH}$ & $3.0671 \pm 0.30$ & $3.2186 \pm 0.55$ & $2.1979 \pm 0.15$ & $<0.05$ & Citrate-EDTA; Heparin-EDTA \\
\hline \multicolumn{7}{|c|}{ Recovery $(\% \pm \mathrm{SD})$} \\
\hline 1 & QCL & $68.27 \pm 8.31$ & $70.49 \pm 5.64$ & $45.20 \pm 1.61$ & $<0.05$ & There is significant difference \\
\hline 2 & $\mathrm{QCH}$ & $64.43 \pm 6.17$ & $69.45 \pm 9.51$ & $49.17 \pm 5.23$ & $<0.05$ & There is significant difference \\
\hline \multicolumn{7}{|c|}{ Stability in $\pm 25^{\circ} \mathrm{C}$ (short-term stability) } \\
\hline 1 & QCL & Minimum $24 \mathrm{~h}$ & Minimum $24 \mathrm{~h}$ & Minimum $24 \mathrm{~h}$ & - & - \\
\hline 2 & $\mathrm{QCH}$ & Minimum $24 \mathrm{~h}$ & Minimum $24 \mathrm{~h}$ & Minimum $24 \mathrm{~h}$ & - & - \\
\hline \multicolumn{7}{|c|}{ Stability post-preparation (autosampler) } \\
\hline 1 & QCL & Minimum $24 \mathrm{~h}$ & Minimum $24 \mathrm{~h}$ & Minimum $24 \mathrm{~h}$ & - & - \\
\hline 2 & $\mathrm{QCH}$ & Minimum $24 \mathrm{~h}$ & Minimum $24 \mathrm{~h}$ & Minimum $24 \mathrm{~h}$ & - & - \\
\hline \multicolumn{7}{|c|}{ Freeze-thaw stability } \\
\hline 1 & QCL & Minimum 3 cycles & Minimum 3 cycles & Minimum 3 cycles & - & - \\
\hline 2 & QCH & Minimum 3 cycles & Minimum 3 cycles & Minimum 3 cycles & - & - \\
\hline \multicolumn{7}{|c|}{ Stability in temperature $-20^{\circ} \mathrm{C}$ (Long-term stability) } \\
\hline 1 & QCL & Minimum 28 days & Minimum 21 days & Minimum 21 days & - & - \\
\hline 2 & $\mathrm{QCH}$ & Minimum 28 days & Minimum 21 days & Minimum 21 days & - & - \\
\hline
\end{tabular}

LLOQ: Lower limit of quantitation, EDTA: Ethylenediaminetetraacetic acid, SD: Standard deviation, QCL: Quality control low, QCM: Quality control medium, QCH: Quality control high

\section{Comparison of the recovery values of all three plasmas}

Observations were made statistically on the concentrations of quality control low (QCL) and Quality control high (QCH). At the concentration of QCL and QCH for the recovery for the three types of plasma, obtained a $\mathrm{p}<0.05$, indicating a significant difference. In the comparative analysis of the recovery values obtained statistically for the concentration of QCL and QCH, $p<0.05$ also indicated significant differences. This may be due to the very small concentrations of the analysis, which were in the picogram per milliliter range, thus the recovery of each plasma varied.

Comparison of clopidogrel stability for all three plasma samples Based on the results of the study (Table 1), clopidogrel was stable in plasma citrate, plasma heparin, and plasma EDTA for short-term storage and autosampler. For long-term stability stored at $-20^{\circ} \mathrm{C}$, clopidogrel was stable for at least 21 days in all three types of plasma. Based on the stability data, there were no significant differences in the stability of clopidogrel in plasma citrate, heparin, and EDTA.

\section{CONCLUSION}

The analytical method met the requirements based on EMEA guidelines from 2011 for all three types of plasma (citrate, heparin, and EDTA). In addition, clopidogrel analysis in plasma citrate or heparin provides better results than in plasma EDTA.

\section{ACKNOWLEDGMENT}

This research was supported by Indexed International Publication for Final Assignment (PITTA) Research Grants 2017 from Universitas Indonesia.

\section{CONFLICTS OF INTEREST}

All authors have none to declare.

\section{REFERENCES}

1. Rodak BF, Fritsma GA, Keohane EM. Hematology Clinical Principles and Applications. Hematology Clinical Principles and Applications. Missouri: Elseviers; 2012.

2. Wells BG, DiPiro JT, Schwinghammer TL, DiPiro CV. Pharmacotherapy Handbook. $9^{\text {th }}$ ed. New York: McGraw-Hill; 2015.
3. Lüllmann H, Mohr K, Hein L, Bieger D. Color Atlas of Pharmacology. $3^{\text {rd }}$ ed. New York: Georg Thieme Verlag; 2005.

4. Lainesse A, Ozalp Y, Wong H, Alpan RS. Bioequivalence study of clopidogrel bisulfate film-coated tablets. Arzneimittel Forschung 2004;54:600-4.

5. Bowen RA, Hortin GL, Csako G, Otañez OH, Remaley AT. Impact of blood collection devices on clinical chemistry assays. Clin Biochem 2010;43:4-25.

6. Barri T, Dragsted LO. UPLC-ESI-QTOF/MS and multivariate data analysis for blood plasma and serum metabolomics: Effect of experimental artefacts and anticoagulant. Anal Chim Acta 2013;768:118-28.

7. Li W, Zhang J, Tse FL, Li W, Zhang J, Tse FL, editors. Handbook of LC-MS Bioanalysis : Best Practices, Experimental Protocols, and Regulations. New Jersey: John Wiley and Sons; 2013.

8. Moffat AC, Osselton M, Widdop B, Moffat AC, Osselton M, Widdop B, editors. Clark's Analysis of Drugs and Poisons. $3^{\text {rd }}$ ed. London: Pharmaceutical Press; 2004

9. Christensen JM, Stalker D. Ibuprofen piconol hydrolysis in vitro in plasma, whole blood, and serum using different anticoagulants. J Pharm Sci 1991;80:29-31

10. Barton RH, Waterman D, Bonner FW, Holmes E, Clarke R, Procardis Consortium, et al. The influence of EDTA and citrate anticoagulant addition to human plasma on information recovery from NMR-based metabolic profiling studies. Mol Biosyst 2010;6:215-24.

11. Evans G. A Handbook of Bioanalysis and Drug Metabolism. A Handbook of Bioanalysis and Drug Metabolism. Boca Raton: CRC Press; 2004.

12. Yi J, Craft D, Gelfand CA. Minimizing preanalytical variation of plasma samples by proper blood collection and handling. Methods Mol Biol 2011:728:137-49.

13. Gonzalez-Covarrubias V, Dane A, Hankemeier T, Vreeken RJ. The influence of citrate, EDTA, and heparin anticoagulants to human plasma LC-MS lipidomic profiling. Metabolomics 2013;9:337-48.

14. Harahap Y. The Role of Bioanalysis in Drug Quality Assurance and Improving Quality of Patient Life. Jakarta: Universitas IndonesiaPress; 2010

15. Hall TG, Smukste I, Bresciano KR, Wang Y, Mckearn D, Savage RE. Identifying and overcoming matrix effects in drug discovery and development. In: Tandem Mass Spectrometry-Applications and Principles. INTECH; 2012. p. 794.

16. Harahap Y, Masyarah I, Suryadi H. Analytical validation of clopidogrel in human plasma through ultra-high performance liquid chromatographytandem mass spectrometry. Int J App Pharm 2017;9:721-33.

17. Waters. Waters UPLC, UHPLC, and HPLC Column Selection and Mobile Phase Guide Wide pH Range. USA: Waters Corporation; 2016.

18. Shaikh S, Jain V. A novel reverse-phase high-performance liquid 
chromatographic method for simultaneous estimation of ellagic acid, quercetin, and piperine in ayurvedic formulations. Asian J Pharm Clin Res 2018;11:312-7.

19. Rao N, Gawde K. Method development and force degradation studies for simultaneous estimation of salbutamol sulfate, etofylline and bromhexine hydrochloride in pharmaceutical dosage form using reversed-phase high-performance liquid chromatography method. Asian J Pharm Clin Res 2018;11:378-82.
20. Selvaraj NA, Vijayalkshmi K. Chromatographic separation of bioactive compounds from ipomoeae batatas lam by Column, HPTLC and GCMS techniques. Asian J Pharm Clin Res 2014;7:4-8.

21. Fountain KJ. UPLC Versus UHPLC : Comparison of Loading and Peak Capacity for Small Molecule Drugs. Milford, USA: Waters Corporation; 2011. p. 1-6.

22. European Medicines Agency. Guideline on the Investigation of Bioequivalence. Vol 1. London: European Medicines Agency; 2010. 